\title{
Bortezomib Rescues Ovariectomy-Induced Bone Loss via SMURF- Mediated Ubiquitination Pathway
}

\author{
Yuepeng Fang, ${ }^{1,2,3}$ Yang Liu,, ${ }^{1,2}$ Zhijian Zhao,,2 Yingjie Lu, ${ }^{1,2}$ Xu Shen,, ${ }^{1,2}$ Tianfeng Zhu, ${ }^{1,2}$ \\ Mingzhuang Hou, ${ }^{1,2}$ Fan He, ${ }^{1,2}$ Huilin Yang, ${ }^{1,2}$ Yijian Zhang $\mathbb{D}^{1,2}$ Qin Shi ${ }^{1 D},{ }^{1,2}$ \\ and Xuesong Zhu $\mathbb{D}^{1,2}$ \\ ${ }^{1}$ Department of Orthopaedics, The First Affiliated Hospital of Soochow University, Soochow University, Suzhou 215006, China \\ ${ }^{2}$ Orthopaedic Institute, Medical College, Soochow University, Suzhou 215007, China \\ ${ }^{3}$ Jinan Central Hospital, Cheeloo College of Medicine, Shandong University, No. 105, Jiefang Road, Jinan, Shandong 250013, China
}

Correspondence should be addressed to Yijian Zhang; yjzhang2013@stu.suda.edu.cn, Qin Shi; shiqin@suda.edu.cn, and Xuesong Zhu; zhuxs@suda.edu.cn

Yuepeng Fang, Yang Liu, Zhijian Zhao, and Yingjie Lu contributed equally to this work.

Received 14 September 2021; Revised 17 November 2021; Accepted 29 November 2021; Published 31 December 2021

Academic Editor: Antonio Desmond McCarthy

Copyright () 2021 Yuepeng Fang et al. This is an open access article distributed under the Creative Commons Attribution License, which permits unrestricted use, distribution, and reproduction in any medium, provided the original work is properly cited.

\begin{abstract}
A balance between bone formation by osteoblasts and bone resorption by osteoclasts is necessary to maintain bone health and homeostasis. As a cancer of plasma cells, multiple myeloma (MM) is accompanied with rapid bone loss and fragility fracture. Bortezomib has been used as a first-line for treating MM for decades. Recently, the potential protection of bortezomib on osteoporosis (OP) is reported; however, the specific mechanism involving bortezomib-mediated antiosteoporotic effect is undetermined. In the present study, we assessed the effects of in vitro bortezomib treatment on osteogenesis and osteoclastogenesis and the protective effect on bone loss in ovariectomized (OVX) mice. Our results indicated that bortezomib treatment increased osteogenic differentiation of MC3T3-E1 cells as evidenced by increased levels of matrix mineralization and osteoblast-specific markers. In bortezomib-treated bone marrow monocytes (BMMs), osteoclast differentiation was suppressed, substantiated by downregulated tartrate-resistant acid phosphatase- (TRAP-) positive multinucleated cells, areas of actin rings, pit formation, and osteoclast-specific genes. Mechanistically, bortezomib exerted a protective effect against OP through the Smad ubiquitination regulatory factor- (SMURF-) mediated ubiquitination pathway. Furthermore, in vivo intraperitoneal injection of bortezomib attenuated the bone microarchitecture in OVX mice. Accordingly, our findings corroborated that bortezomib might have future applications in the treatment of postmenopausal OP.
\end{abstract}

\section{Introduction}

Osteoporosis (OP) is a progressive metabolic disease manifested as degeneration of bone structure and impaired bone mass [1]. Approximately 71.8 million people over 50 years suffer from OP in China, a 25-fold higher prevalence than in the United Kingdom [2]. Severe OP is accompanied by increased bone fragility, leading to increased risks of osteoporotic vertebral compression fracture (OVCF) and hip fracture [3], associated with considerable morbidity and mortality. Mechanically, although OP can be divided into two subtypes: estrogen-deficient OP and senile OP based on the etiology, the collective driving factor is disrupted homeostasis between bone formation and bone resorption [4]. An osteoblast/osteocyte-induced osteogenesis shift toward overactivated osteoclast-mediated osteoclastogenesis can lead to excessive bone loss and microarchitectural impairment [5]. Therefore, targeting the regulation of bone metabolism is essential for reversing the progression of OP.

Bortezomib is a boronic acid dipeptide first reported as an antineoplastic agent for multiple myeloma (MM) [6]. Bortezomib exerts anticancer effects during oncologic 
therapy by inhibiting proteasome [7], inducing apoptosis and cell cycle arrest. Recently, bortezomib has been used in the treatment of non-hematologic malignancies, including degenerative musculoskeletal diseases (osteoarthritis, OA) [8], rheumatoid arthritis (RA) [9], and titanium particleinduced osteolysis [10]. Accumulating data indicate that during MM treatment, bortezomib also affects bone metabolism [11]. Suominen et al. reported that in vivo combination treatment of bortezomib and radium-223 alleviated myeloma-induced bone destruction in the syngeneic 5TGM1 MM mice model [12]. Furthermore, in mice models of ovariectomy-induced OP, intraperitoneal injection of bortezomib delayed the bone loss by inhibiting bone resorption [13]. However, the underlying mechanisms by which bortezomib modulates bone homeostasis are far from being known.

As a posttranslational modification, ubiquitination has been documented in multiple biological processes [14]. A classical ubiquitin-proteasome system (UPS) consists of three main factors: ubiquitin-activating enzyme (E1), conjugating enzyme (E2), and protein ligase (E3) [15]. Interestingly, bone metabolism can be modulated by the ubiquitination of transforming growth factor- (TGF-) $\beta$ and bone morphogenetic protein (BMP) signaling pathways via Smad ubiquitination regulatory factors (SMURFs) [16]. SMURF1 promoted myogenic differentiation but blocked BMP2-mediated osteogenic conversion by activating the ubiquitin degradation of Smad5 in mouse C2C12 myoblast cells [17]. Moreover, a small skeletal phenotype and activated bone resorption were found in Smurf $2^{-7}$ mice with increased ubiquitin levels of Smad1/5 [18]. Intriguingly, Wang et al. demonstrated that bortezomib prevented prostate cancer oncogenesis and bone metastasis by modulation of ubiquitination regulatory factors SMURF1 and SMURF2 [19]. Nevertheless, the mechanisms involving bortezomibinduced ubiquitination in osteoporosis are not elucidated.

Our study was aimed at exploring the effect of in vitro bortezomib treatment on osteoblast and osteoclast differentiation. The protection of bortezomib on preventing bone loss in ovariectomized- (OVX-) impaired mice was also determined. In addition, the potential mechanisms underlying the bortezomib-induced antiosteoporotic effect were explored by targeting SMURF-mediated ubiquitination.

\section{Materials and Methods}

2.1. Cell Culture and Bortezomib Treatment. The osteoblastic cell line MC3T3-E1 was purchased from the Cell Bank of the Chinese Academy of Sciences (Shanghai, China). MC3T3E1 cells were cultured in a medium containing alpha minimum essential medium ( $\alpha$-MEM) (Thermo Fisher Scientific, Waltham, MA, USA), 10\% fetal bovine serum (FBS), and $1 \%$ penicillin and streptomycin at $37^{\circ} \mathrm{C}$ with $5 \% \mathrm{CO}_{2}$. For osteogenic differentiation, MC3T3-E1 cells were seeded in 12well plates and cultured with an osteogenic differentiation medium $(\alpha$-MEM supplemented with $10 \%$ FBS, $50 \mu \mathrm{g} / \mathrm{mL}$ ascorbic acid, $10 \mathrm{mM} \beta$-glycerophosphate, and $100 \mathrm{nM}$ dexamethasone). As previously described, bone marrow monocytes (BMMs) were isolated from $\mathrm{C} 57 \mathrm{BL} / 6 \mathrm{~J}$ mice
[20]. Briefly, bone marrow cells were flushed from the tibias and femurs of mice using a syringe. The marrow cells were incubated with red blood cell lysis buffer (Beyotime, Nantong, China) to remove red blood cells (RBC). Cells were cultured in $10 \mathrm{~cm}$ dishes with a standard growth medium ( $\alpha$-MEM supplemented with $10 \%$ FBS and $1 \%$ penicillin and streptomycin) at $37^{\circ} \mathrm{C}$ with $5 \% \mathrm{CO}_{2}$. The suspended cells were collected and treated with $100 \mathrm{ng} / \mathrm{mL}$ macrophage-colony stimulating factor (M-CSF) (R\&D Systems, Minnesota, USA). For the induction of osteoclast differentiation, BMMs were seeded in 12-well plates and incubated with $\alpha$-MEM with $50 \mathrm{ng} / \mathrm{mL}$ receptor activator of nuclear factor- $\kappa \mathrm{B}$ ligand (RANKL) and $30 \mathrm{ng} / \mathrm{mL} \mathrm{M-}$ CSF (R\&D Systems). To determine the in vitro effect of bortezomib (Sigma-Aldrich) on both osteoblast and osteoclast differentiation, cells were divided into three groups: negative control (NC) group, induced differentiation group, and $1 \mathrm{nM}$ of bortezomib-treated group.

2.2. Alkaline Phosphatase Staining. After 7 days of osteogenic differentiation, MC3T3-E1 cells were washed with phosphate-buffered saline (PBS) and fixed with 4\% paraformaldehyde. The cells were stained with BCIP/NBT Alkaline Phosphatase (ALP) Color Development Kit (Beyotime) for $30 \mathrm{~min}$ at room temperature. After washing with PBS, images were captured using an Olympus IX51 microscope (Olympus Corporation, Tokyo, Japan). ALP activity was measured using an ALP assay kit (Beyotime) and normalized to the protein concentration.

2.3. Alizarin Red S Staining. After 14 days of osteogenic differentiation, MC3T3-E1 cells were fixed with 4\% paraformaldehyde for $15 \mathrm{~min}$. The cells were then incubated with $40 \mathrm{mM}$ Alizarin Red S solution (Sigma-Aldrich, St. Louis, $\mathrm{MO}$, USA) for $30 \mathrm{~min}$ at room temperature. Digit images were observed using an inverted microscope. The stain was extracted with $5 \%$ perchloric acid solution, and the matrix mineralization level was quantified using a spectrophotometer (BioTek).

2.4. F-Actin Staining. BMMs were seeded on a 24-well plate and treated with $50 \mathrm{ng} / \mathrm{mL}$ RANKL and $30 \mathrm{ng} / \mathrm{mL} \mathrm{M-CSF}$ to induce osteoclasts. To determine the effect of bortezomib, cells were treated with $1 \mathrm{nM}$ bortezomib and the culture media were changed every other day. After 5 days of osteoclastogenic differentiation, BMMs were washed with PBS and fixed with $4 \%$ paraformaldehyde. The cells were incubated with $0.1 \%$ Triton X-100 for $20 \mathrm{~min}$ and stained with CytoPainter Phalloidin-iFluor 488 Reagent (Abcam, Cambridge, MA, USA) for $1 \mathrm{~h}$. Cell nuclei were counterstained using a $4^{\prime}, 6$-diamidino-2-phenylindole (DAPI) for $4 \mathrm{~min}$. Immunofluorescence images of F-actin rings were observed with an inverted fluorescence microscope. The number of F-action rings was measured with an ImageJ software.

2.5. Pit Formation. BMMs were seeded on 96-well plates coated with the inorganic bone biomaterial surface (Corning Life Sciences, NY, USA). After 6 days of osteoclast differentiation, cells were removed with a $2 \%$ hypochlorite solution 
TABLE 1: Primers used for quantitative real-time RT-PCR.

\begin{tabular}{lcc}
\hline Gene & Forward primer sequence $\left(5^{\prime}-3^{\prime}\right)$ & Reverse primer sequence $\left(5^{\prime}-3^{\prime}\right)$ \\
\hline Gapdh & GGTGAAGGTCGGTGTGAACG & CTCGCTCCTGGAAGATGGTG \\
Alp & CCAACTCTTTTTGGCCAGAGA & GGCTACATTGGTGTTGAGCTTTT \\
Runx 2 & TCTCCACTCTTCTAGTTGGGAC & TTGGGTCATTTCCACATGC \\
Sp7 & GTGACACCGTGTCAGCAAAG & GGAGCACAGGAACTTGGGAC \\
Bglap & GGAAAGGAGGCACAAAGAAGC & CCCCTTAGGCACTAGGAGC \\
Trap & CTTGGTGCACACCTAGCAGA & CTCCCTCATGTGTTGTCCCT \\
Ctsk & GACCTCCAAGTTCTTATCCTCAC \\
Mmp 9 & GACGCAGCGATGCTAACTAA & ACTGATACCGTCTGTCATCCC \\
Nfatc1 & CTGGACAGCCAGACACTAAAG & CCAGCACAGAGTCCACAACT \\
Smurf 1 & ACAGAGTTACCATTGGCAGGA & CTCGCGGCAAGTCTTCAGAGAAG \\
& GGTGGCACTGCACTCCTAGAAC & GCTTGAGATACCACCTTTCCG \\
& TGCACTAACAACCTGCCGAAAG & GCGCGGACCCAAACTACAAC \\
\end{tabular}

for $10 \mathrm{~min}$ at room temperature. After washing with PBS, the resorption pits were observed under a microscope.

2.6. TRAP Staining. BMMs were fixed using a $4 \%$ paraformaldehyde solution. The cells were stained with an acetatebuffered solution containing naphthol AS-BI phosphate and tartrate (Sigma-Aldrich) at $37^{\circ} \mathrm{C}$ for $1 \mathrm{~h}$. TRAPpositive multinucleated cells were counted and photographed using an Olympus IX51 microscope.

2.7. RT-PCR. Total RNA was extracted using a TRIzol reagent, and $1 \mu \mathrm{g}$ of RNA was used to synthesize cDNA using a cDNA Synthesis Kit. Quantitative real-time RTPCR was performed using an SYBR Green Supermix kit. Transcript levels of Alp, Col1a1, Runx2, Sp7, Bglap, Smurf1, Smurf2, Trap, Ctsk, Mmp9, and Nfatc1, with Gapdh, were used as internal controls. The expression levels of mRNAs were calculated according to a $\Delta \mathrm{Ct}\left(2^{-\Delta \Delta \mathrm{Ct}}\right)$ method. The primer sequences are shown in Table 1.

2.8. Western Blot. Cellular proteins were extracted from osteoclast or osteoblast using a cell lysis buffer (Beyotime) supplemented with proteinase inhibitors (Thermo Fisher Scientific) on ice for $1 \mathrm{~h}$. The extracted protein was subjected to $10 \%$ sodium dodecyl sulfate-polyacrylamide gel (SDSPAGE) followed by transferring electrophoretically onto a nitrocellulose membrane (Beyotime). The membranes were blocked and then incubated with primary antibodies against OPG (Abcam, ab73400), RUNX2 (Cell Signaling Technology, 12556S), OSX (ab209487), OCN (ab13420), SMURF1 (2174S), SMURF2 (12024S), P-SMAD1/5/9 (13820S), CTSK (ab37259), TRAP (ab191406), NFATC1 (8032S), MMP9 (13667S), P-P65 (Abclonal, AP0124), P-ERK (AP1120), and $\beta$-actin (3700S). Subsequently, membranes were incubated with the secondary antibodies for $1 \mathrm{~h}$ at room temperature. Bound antibodies were imaged on a VersaDocTM imaging system (Bio-Rad). After normalization against $\beta$ actin as an internal standard, the relative expression levels of proteins were calculated with an ImageJ Software.

2.9. Bioinformatic Prediction. The GSE156508 dataset containing osteoblast transcriptome from six controls and six osteoporotic patients was downloaded from the National Center for Biotechnology Information (NCBI) search database and Gene Expression Omnibus (GEO) database. Screening of differentially expressed genes (DEGs) and generation of volcano plots and heat maps were performed using R software. Protein and protein interaction (PPI) was conducted using an online analyzing tool STRING (https:// string-db.org/). Gene Ontology (GO) and Kyoto Encyclopedia of Genes and Genome (KEGG) analyses were performed to explore the comprehensive biological process (BP), cellular component (CC), molecular function (MF), and signaling pathways.

2.10. OVX-Induced Bone Loss. Animal experiments were handled according to the protocols of the Ethics Committee of Soochow University. Eight-week-old female mice (C57BL/6J) were provided by the Experimental Animal Center of Soochow University. A total of 24 mice were randomly allocated into three groups (8 per group). After anesthesia with sodium pentobarbital, Group A mice were subjected to a sham operation, while Group B and C mice underwent bilateral ovariectomy. After surgery, mice were injected with penicillin intramuscularly for three days to avoid infection.

2.11. Administration of Bortezomib. Bortezomib was dissolved in dimethylsulfoxide (DSMO) (Sigma-Aldrich) and then diluted in saline for subsequent injection. One week after surgery, Groups A and B received an intraperitoneal injection of $0.9 \%$ saline, while Group $\mathrm{C}$ received an intraperitoneal injection of $0.5 \mathrm{mg} / \mathrm{kg}$ bortezomib. All injections were performed twice a week for six weeks. Mice were anesthetized with isoflurane and then euthanized using the cervical dislocation method eight weeks after surgery, and bilateral femurs were collected for the following analyses.

2.12. Microcomputed Tomography $(\mu C T)$ Analysis. The bone mass of distal femurs was evaluated using a $\mu \mathrm{CT}$ system with a high resolution $(16 \mu \mathrm{m})$ at $60 \mathrm{kV}(380 \mu \mathrm{A})$. Data reconstruction was performed using NRecon v1.6 software. Regions of interest (ROI) were drawn $500 \mu \mathrm{m}$ from the end of the epiphyseal growth plate to points $1 \mathrm{~mm}$ along the 

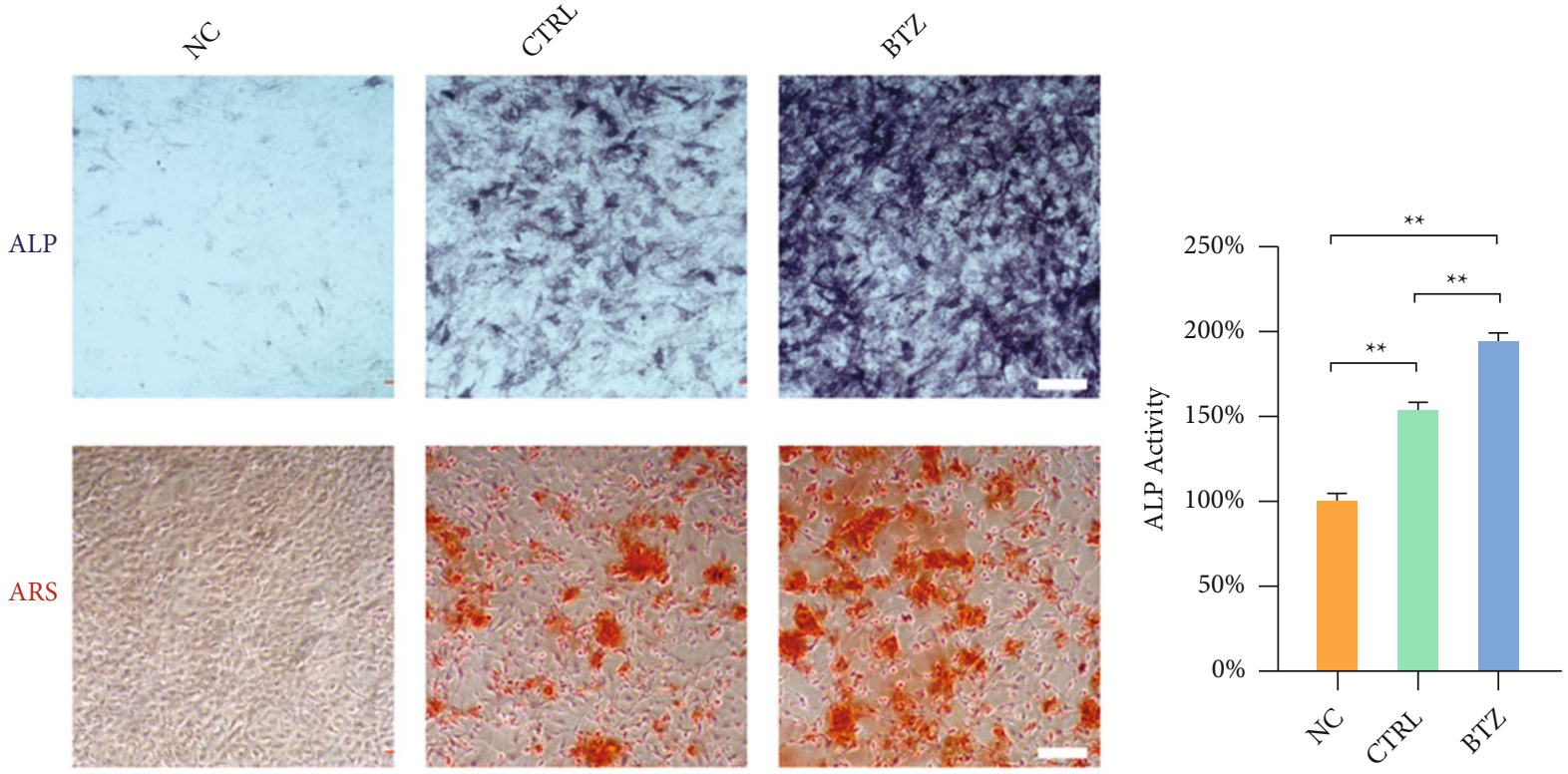

(a)

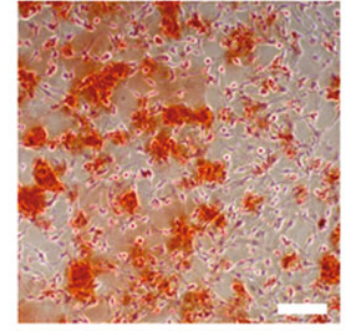

(b)
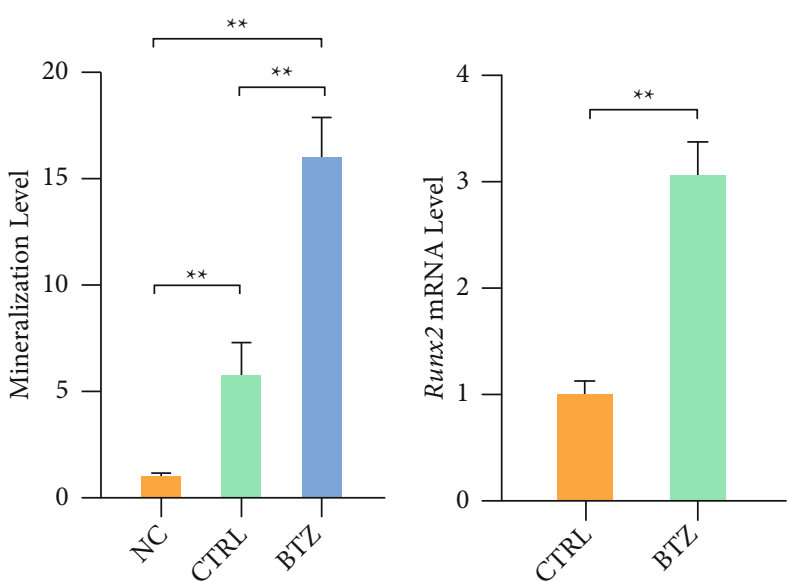

(c)

(d)
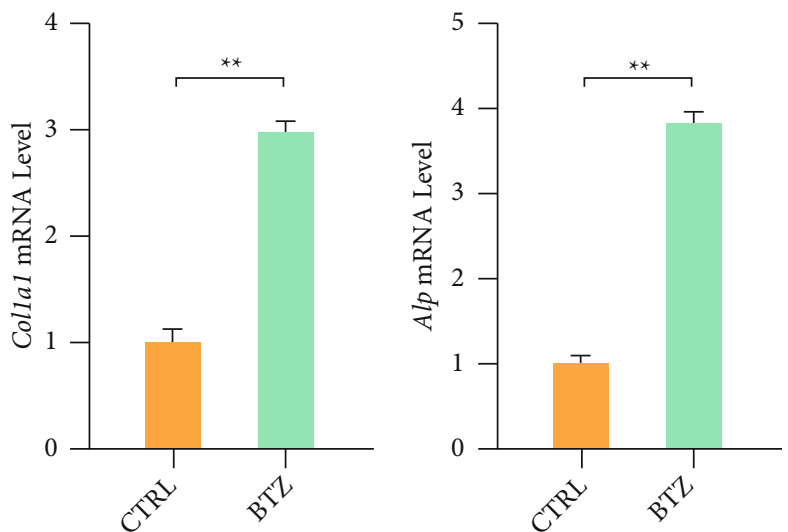

(e)

(f)

Figure 1: Continued. 


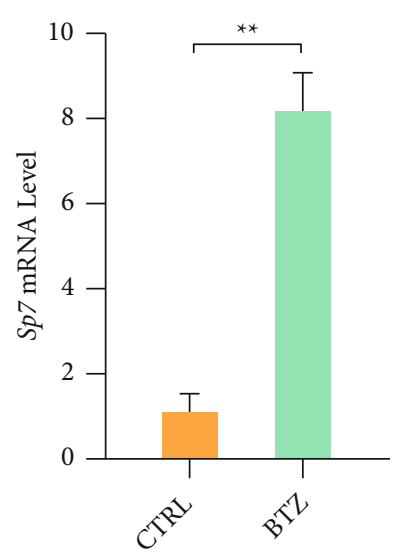

(g)

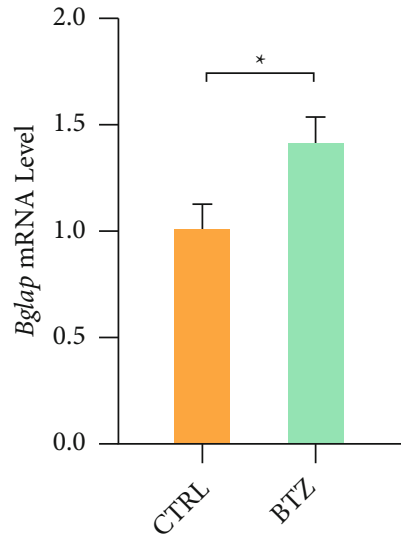

(h)

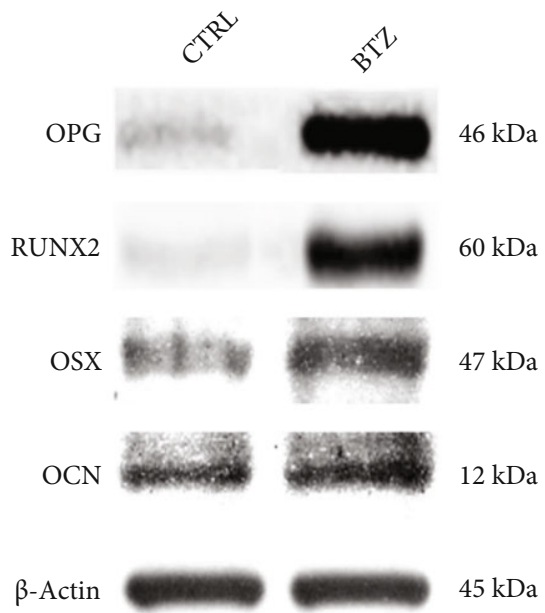

(i)

FIGURE 1: The effect of bortezomib on the osteogenic differentiation of MC3T3-E1 cells. (a) Representative images of mineralized extracellular matrix stained by Alkaline Phosphatase and Alizarin Red S staining. Scale bar $=200 \mu \mathrm{m}$. (b) Quantification of ALP activity in bortezomib-treated MC3T3-E1 cells. (c) Quantification of stained mineral layers in bortezomib-treated MC3T3-E1 cells. The values were normalized to the level of the NC group. (d-h) The mRNA levels of osteoblast-specific marker genes, including Runx2, Colla1, Alp, Sp7, and Bglap were quantified with real-time RT-PCR using GAPDH as the normalization. (i) The protein levels of osteoblast-specific marker enzymes including OPG, RUNX2, OSX, and OCN were determined using Western blot assays. Values represent mean \pm SD of three replicas for ALP activity and ARS staining assays, three replicas for RT-PCR experiments and three replicas for Western blot assays, respectively. Statistically significant differences are indicated by $*$ where $p<0.05$ or $* *$ where $p<0.01$ between the indicated groups.

cortical bone. The bone microarchitectural parameters, including bone mineral density (BMD), the bone volume ratio (BV/TV), bone surface/volume ratio (BS/BV), and trabecular number (Tb.N), were calculated.

2.13. Histology and Immunohistochemistry (IHC). Femurs were fixed in formalin and then decalcified in Ethylenediaminetetraacetic Acid (EDTA) buffer (Yuanye) for four weeks. Bones were then dehydrated with $50 \%, 70 \%, 80 \%$, and $90 \%$ ethanol and embedded in paraffin (Thermo Fisher Scientific). Longitudinal sections at $5 \mu \mathrm{m}$ thickness were cut and stained with hematoxylin and eosin ( $\mathrm{H} \& \mathrm{E})$ and TRAP staining. Osteoblast areas were evaluated with $\mathrm{H} \& \mathrm{E}$ staining, and osteoclast numbers were counted in TRAP-stained sections using ImageJ software. For immunohistochemical staining, the slides were retrieved using testicular hyaluronidase for 45 minutes. After blocking with 5\% goat serum, the slides were incubated with anti-SMURF, anti-SMURF2, anti-OCN (23418-1-AP), and anti-p-SMAD1/5/9 primary antibodies at $4^{\circ} \mathrm{C}$ overnight. Then, the slides were incubated with a second antibody for $2 \mathrm{~h}$. As previously described, the staining was performed using a 3,3'-Diaminobenzidine (DAB) method [21]. Digit images were observed under a bright-field microscope.

2.14. Statistical Analysis. Data are presented as the mean \pm standard deviation (SD). Statistical analyses were conducted using an independent two-tailed Student's $t$-test for two groups or one-way Analysis of Variance (ANOVA) with Tukey's post hoc test for multiple groups using a GraphPad Prism 8. A probability value of $p$ less than 0.05 was considered statistically significant.

\section{Results}

3.1. In Vitro Bortezomib Treatment Enhances the Osteogenesis of MC3T3-E1 Cells. After 7 days of osteogenic induction, the number of ALP-positive cells was higher in the bortezomib-treated group than in the free-treated cells (Figure 1(a)). Quantitative analysis indicated that bortezomib treatment increased the ALP activity in MC3T3-E1 cells by $26.5 \%$ (Figure 1(b)). After 2 weeks of osteogenic differentiation, Alizarin Red S staining revealed that bortezomib treatment promoted calcium deposits in the matrix of MC3T3-E1 cells. The level of calcium deposits was increased by 1.8 -folds in the bortezomib-treated group (Figure 1(c)). RT-PCR revealed that, after 2 weeks of osteogenic induction, bortezomib treatment upregulated the mRNA expressions of Runx2 by $207.1 \%$, Colla 1 by $197.6 \%$, Alp by $282.9 \%$, Sp7 by $718.0 \%$, and Bglap by $41.6 \%$ in comparison with the freetreated group (Figures $1(\mathrm{~d})-1(\mathrm{~h})$ ). Western blot confirmed that the protein levels of osteogenesis were consistent with their transcript levels (Figure 1(i) and Fig. S1A-C).

3.2. In Vitro Bortezomib Treatment Suppresses Osteoclast Differentiation of BMMs. The TRAP staining showed that bortezomib treatment inhibited osteoclastogenesis in BMMs (Figure 2(a)). Quantitative results indicated the inhibition of the number and the area of the TRAP-positive cells in the bortezomib-treated group (Figure 2(b) and Fig. S2A). The administration of bortezomib suppressed the formation of F-actin rings as observed by the decreased number and area (Figure 2(c) and Fig. S2B). Similarly, osteoclast-induced bone resorption on the bone slices was restrained with the treatment of bortezomib (Fig. S2C). Furthermore, after five days of osteoclastogenic induction, bortezomib treatment 

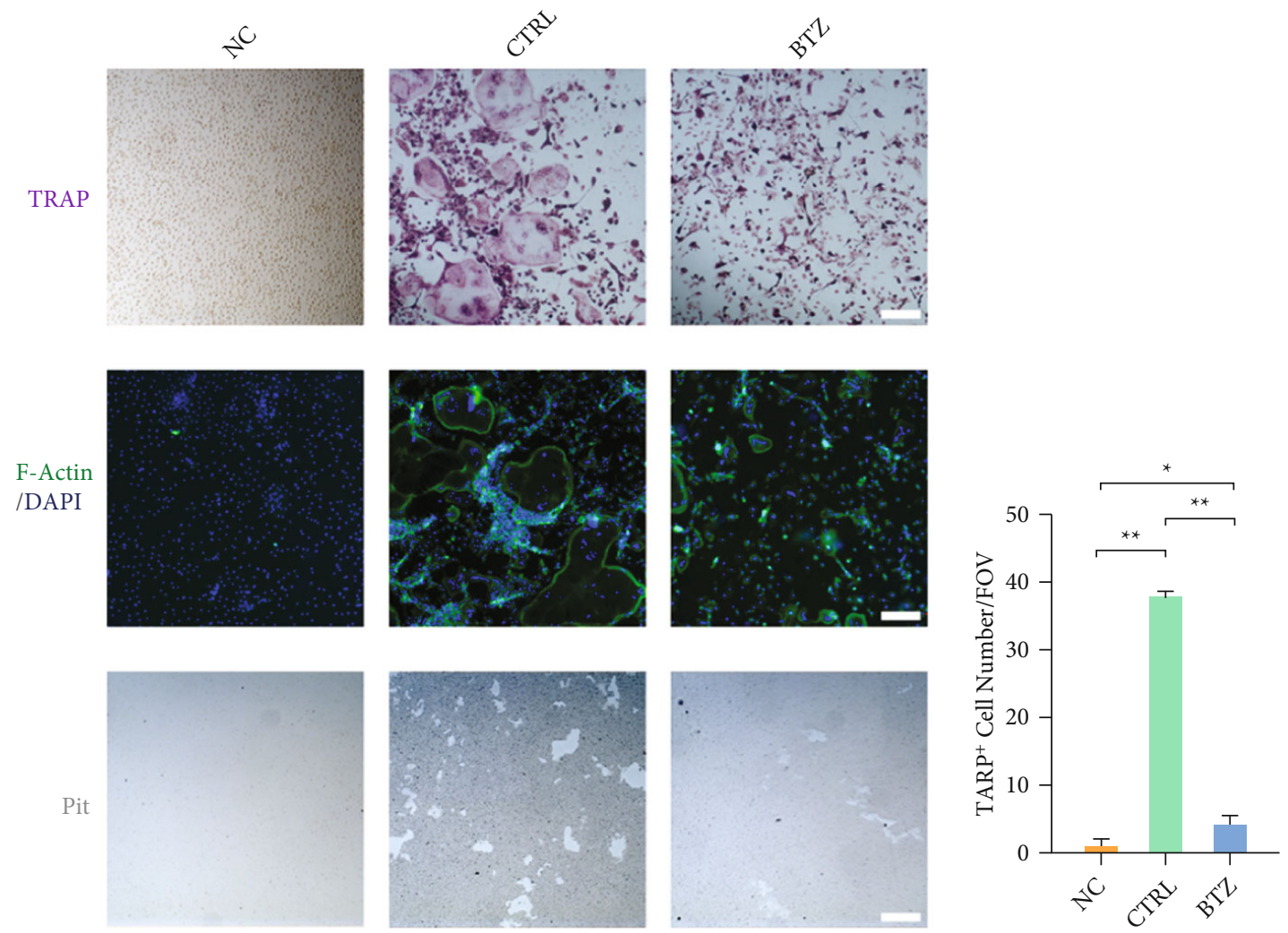

(a)

(b)
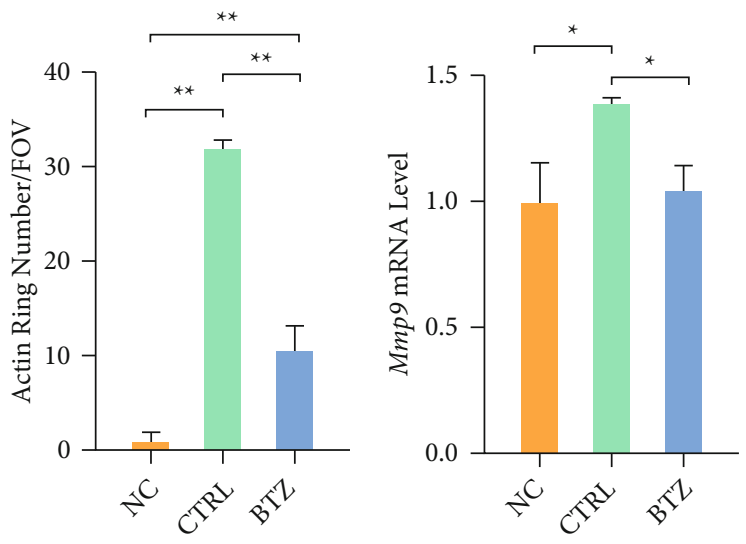

(c)

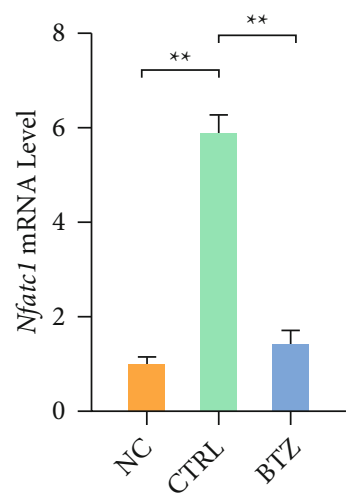

(d)

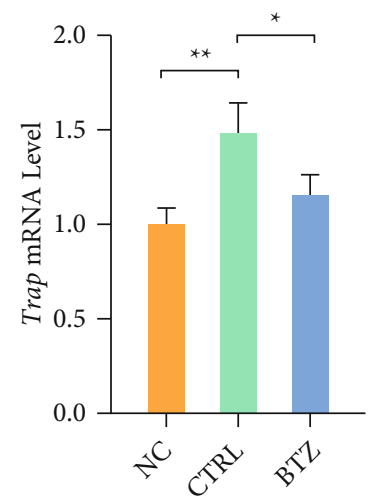

(e)

Figure 2: Continued. 

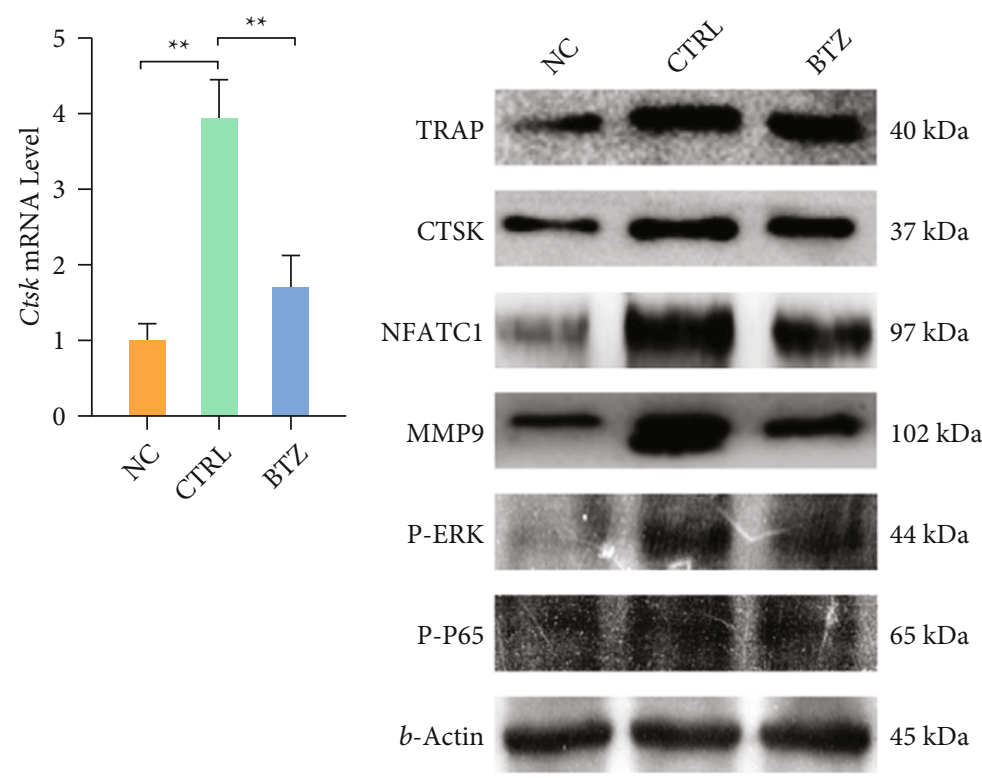

(g)

(h)

FIGURE 2: The effect of bortezomib on the osteoclastogenic differentiation of BMM cells. (a) Representative images of multinucleated osteoclasts stained by TRAP, F-actin rings, and pit formation. Scale bar $=200 \mu \mathrm{m}$. (b) Quantification of the cell number of TRAP-positive osteoclasts in randomly chosen fields of view (FOV) of the bortezomib-treated BMM cells. (c) Quantification of the number of F-actin rings in randomly chosen FOV of the bortezomib-treated BMM cells. (d-g) The mRNA levels of osteoclast-specific marker genes, including Trap, Ctsk, Nfatc1, and Mmp9 were quantified with real-time RT-PCR using GAPDH as the normalization. (h) The protein levels of osteoblast-specific marker enzymes were determined using Western blot assays. Values represent mean \pm SD of three replicas for TRAP and F-actin staining assays, three replicas for RT-PCR experiments and three replicas for Western blot assays, respectively. Statistically significant differences are indicated by $*$ where $p<0.05$ or $* *$ where $p<0.01$ between the indicated groups.

decreased the transcript levels of Trap by $28.2 \%$, Ctsk by $135.2 \%$, Nfatc1 by $307.3 \%$, and $M m p 9$ by $34.2 \%$ (Figures $2(\mathrm{~d})-2(\mathrm{~g}))$. Western blot confirmed that in vitro treatment with bortezomib inhibited the protein levels of osteoclast differentiation markers, as well as restrained the ERK-MAPK and NF- $\kappa$ B signaling pathways (Figure 2(h) and Fig. S2D-I).

\subsection{Bioinformatic Analysis between Control and Osteoporotic} Osteoblast. We performed a bioinformatic analysis on the GEO dataset GSE156508 consisting of osteoblast transcriptome data from diseased samples and normal controls to explore the mechanisms underlying bortezomib-induced bone protection. A total of 48 DEGs, of which 17 were upregulated, and 31 were downregulated, were screened from the control and osteoporosis groups (Figure 3(a)). A heat map revealed that the transcript levels of Smurf2 were decreased by $81.2 \%$, Rgs 4 was decreased by $98.2 \%$, and Hey 2 was increased by 77.6 -folds in the OP group (Figure 3(b)). GO enrichment showed that cell communication, plasma membrane, and cell adhesion molecule activity were key biological behaviors in OP progression (Figure 3(c)). Pathway enrichment analysis revealed that multiple pathways, including mitogen-activated protein kinase (MAPK) signaling pathway (hsa04010), osteoclast differentiation (hsa04380), and ubiquitin-mediated proteolysis (hsa04120), participated in the development of OP (Figure 3(d)).
3.4. Administration of Bortezomib Delays OVX-Induced Bone Loss. To determine the antiosteoporotic effect of bortezomib on estrogen-deficient $\mathrm{OP}, \mathrm{OVX}$ mice received intraperitoneal injection of bortezomib. Eight weeks after bortezomib treatment, $\mu \mathrm{CT}$ analysis showed restored bone loss in the bortezomib-treated group (Figure 4(a)). Quantitatively, administration of bortezomib increased BMD by $25.5 \%, \mathrm{BV} / \mathrm{TV}$ by $66.4 \%, \mathrm{BS} / \mathrm{TV}$ by $72.3 \%$, and Tb.N by $10.5 \%$ (Figures 4(b)-4(e)). Furthermore, histomorphological staining corroborated the bone protection of bortezomib treatment in vivo (Figure $5(\mathrm{a})$ ). The H\&E staining confirmed that the $\mathrm{Tb}$ area was increased by $54.4 \%$ in the bortezomibtreated OVX mice (Figure 5(b)). In vivo TRAP staining revealed that bortezomib treatment reduced the TRAPpositive cells by $50.7 \%$ in OVX mice (Figure $5(\mathrm{c})$ ). The percentage of OCN-positive cells was increased by $26.6 \%$ with bortezomib treatment compared with the OVX mice (Figure 5(d)).

3.5. Bortezomib Exerts Antiosteoporotic Effects via Suppressing SMURF-Mediated SMAD Ubiquitination. Based on the above bioinformatic results, we further investigated the role of SMURF-mediated ubiquitination in the antiosteoporotic effect of bortezomib. RT-PCR showed that bortezomib treatment downregulated the mRNA levels of Smurf1 by $25.9 \%$ and Smurf2 by $56.8 \%$ (Figures $6(\mathrm{a})$ and 6(b)). Western blot revealed that the protein levels of SMURF1 and SMURF2 were decreased in the bortezomib- 


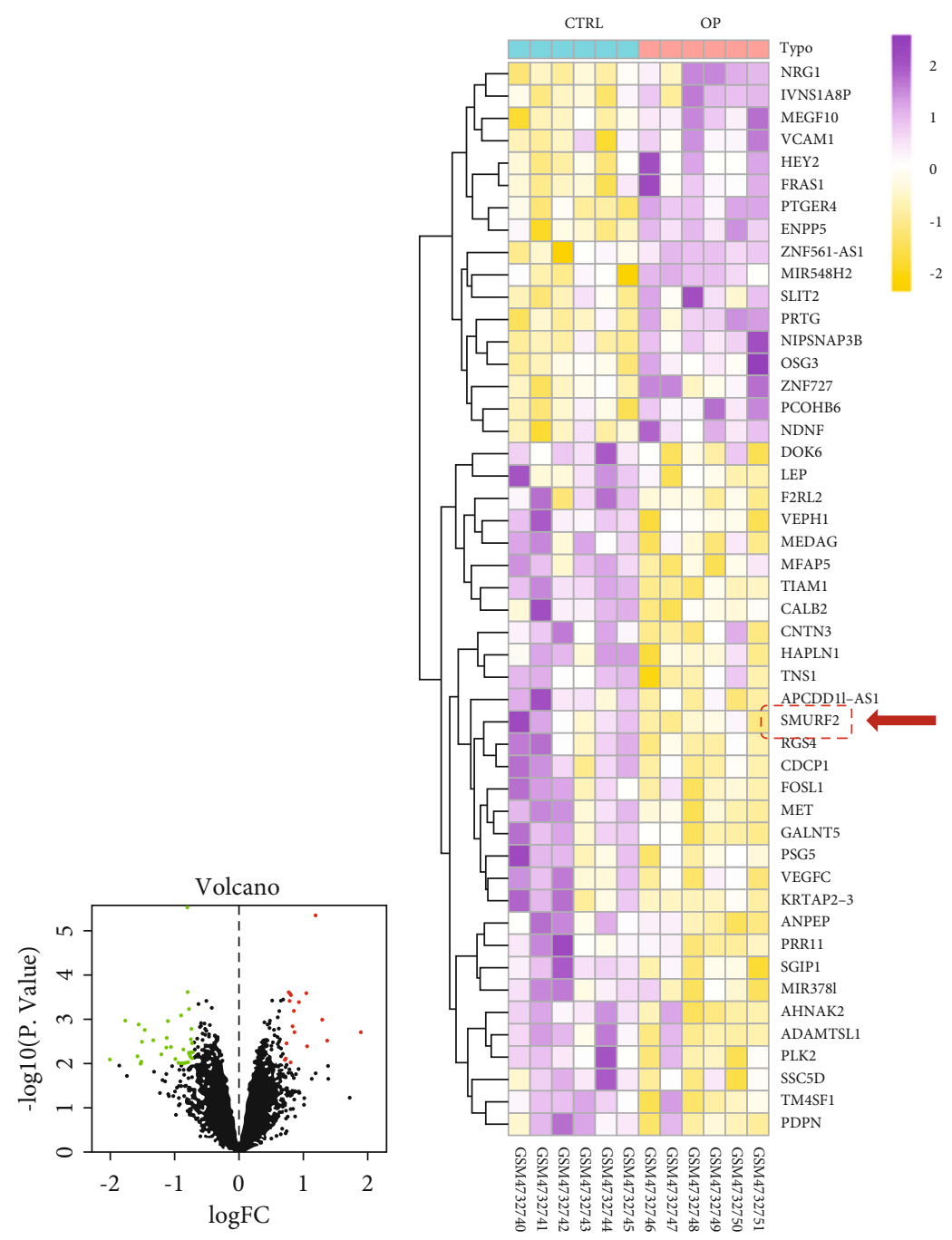

(a)

(b)
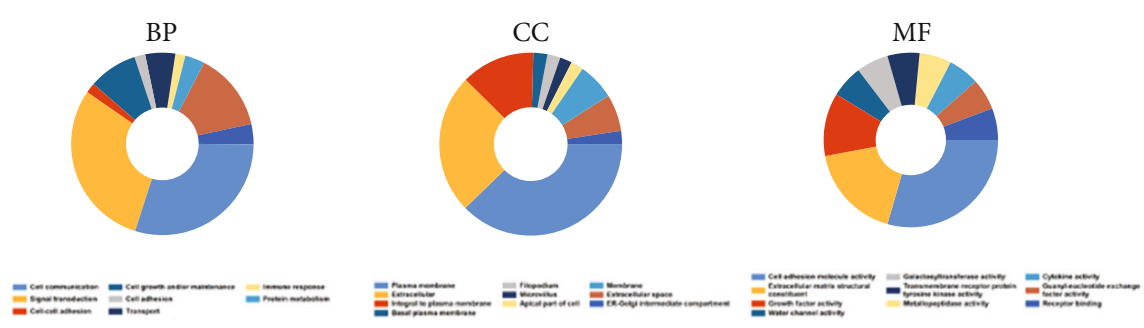

(c)

Figure 3: Continued. 


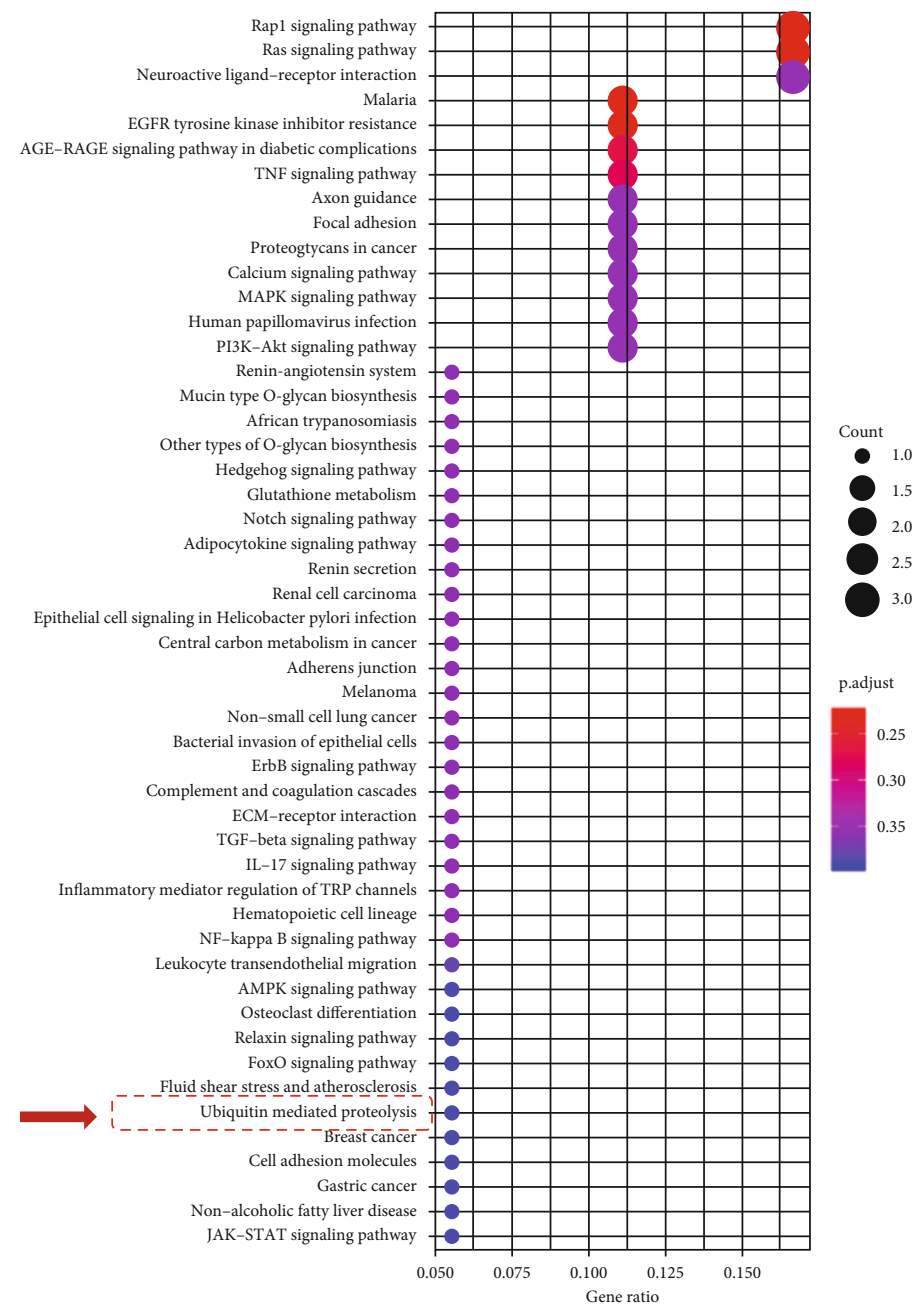

(d)

FIgURE 3: Bioinformatic analysis between the normal and OP patients. (a) Volcano map for the differentially expressed genes between the normal and OP patients. (b) Heat map for the differentially expressed genes between the normal and OP patients. (c) GO enrichment analysis for target genes according to biological process (BP), cellular component (CC), and molecular function (MF). (d) KEGG enrichment analysis for targets genes. Size of the dots (gene count) represents the number of genes. Color of the dots represents the $p$ value.

treated group. However, bortezomib promoted a 1.4-fold activation of the p-SMAD1/5/9 pathway, as evidenced by the Western blot experiment (Figure 6(c) and Fig. S3A-C). Furthermore, the IHC experiments revealed the effects of in vivo administration of bortezomib on the SMURF-mediated ubiquitination pathway (Figure 6(d)). Bortezomib treatment inhibited the expressions of SMURF1 by $43.0 \%$ and SMURF2 by $44.0 \%$ in OVX mice (Figures 6(e) and 6(f)); however, the pSMAD $1 / 5 / 9$ expression level was improved by $88.8 \%$ in bortezomib-treated mice (Figure 6(g)).

\section{Discussion}

Bone remodeling is a physiological process that integrates osteoblast-induced bone formation and osteoclasticmediated bone resorption and maintains skeletal integrity and mineral homeostasis [22]. Under different circumstances, including estrogen deficiency or aging, bone marrow stem cells (BMMSCs) can exhibit lower osteogenic potential and enhanced adipogenesis and osteoclastogenesis [23]. Targeting the dynamic balance between osteoblast and osteoclast differentiation is potentially key to developing new OP treatment methods. Given that the bone not only acts as a rigid structure to support body motion but also acts as the primary site of hematopoiesis, commonly used antihematologic neoplasm agents may be potential antiosteoporotic candidates. Cumulative shreds of evidence indicate that MM-related bone disorder is characterized by an imbalance between bone formation and resorption and disturbance of the RANKL-RANK-osteoprotegerin (OPG) axis [24]. As an MM-targeted drug, bortezomib was first found in the late $20^{\text {th }}$ century and used clinically for over ten years [25]. Past studies suggested that bortezomib could potentially be used for OP treatment based on elevated serum bone-specific alkaline phosphatase levels after bortezomib treatment [26]. Further research provided in vitro and in vivo evidence 

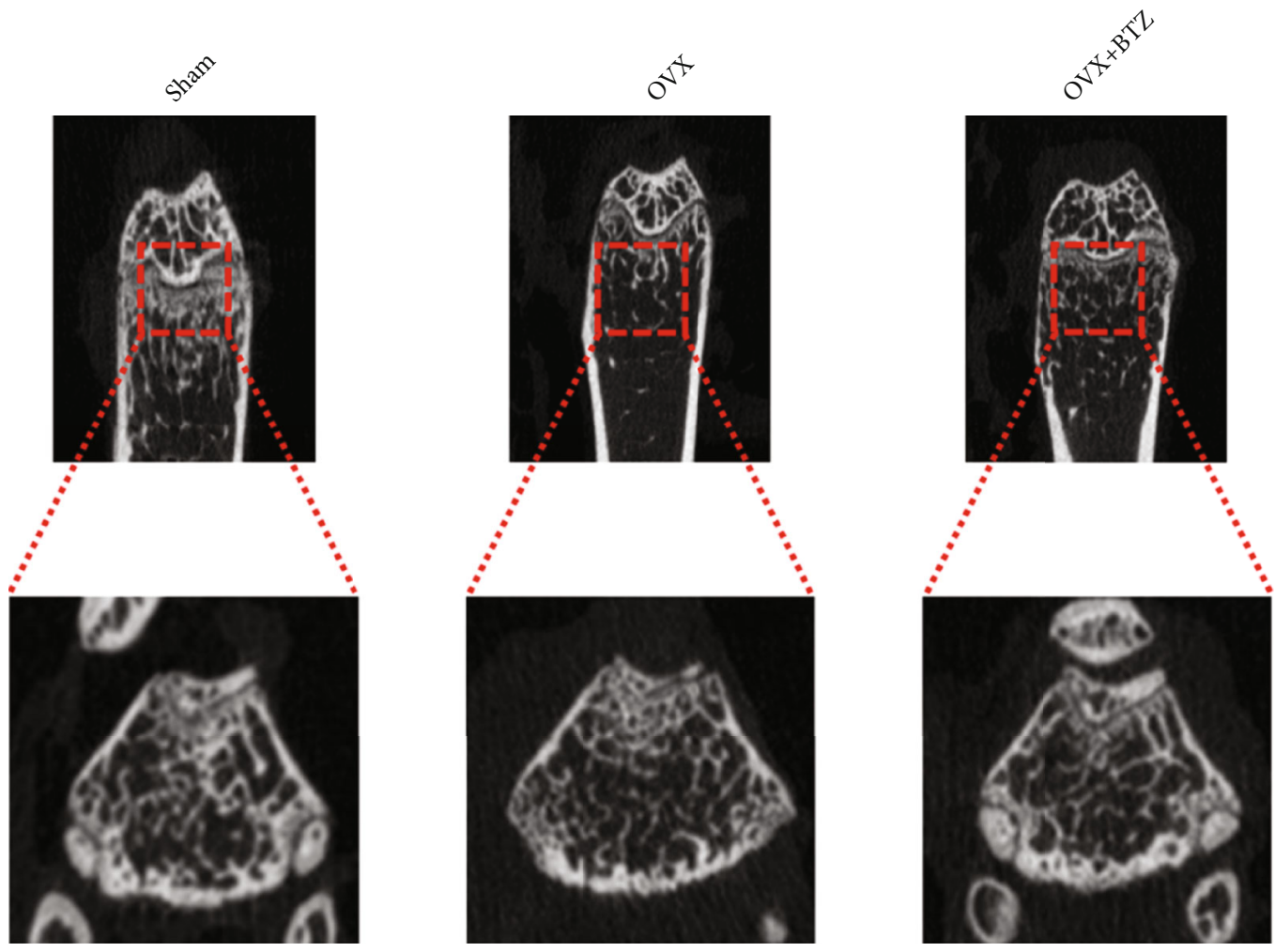

(a)

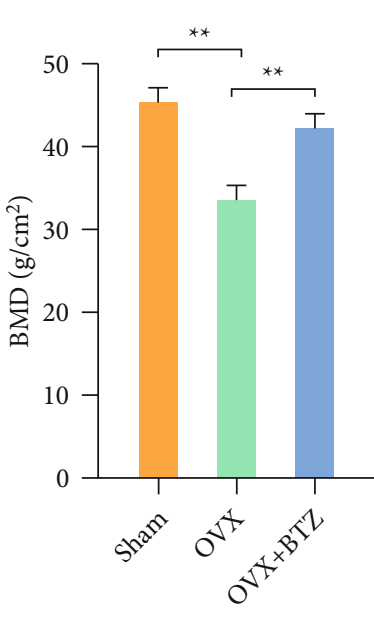

(b)

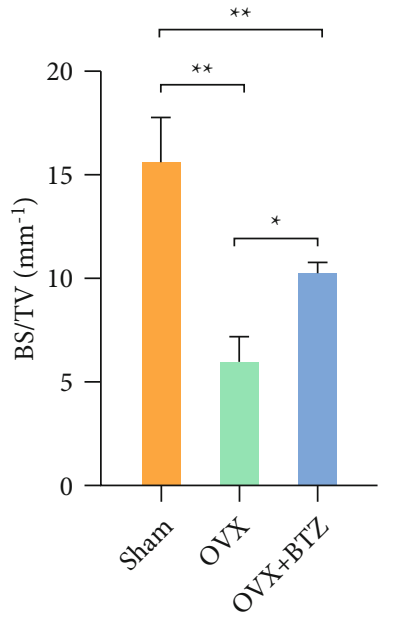

(c)

Figure 4: Continued. 


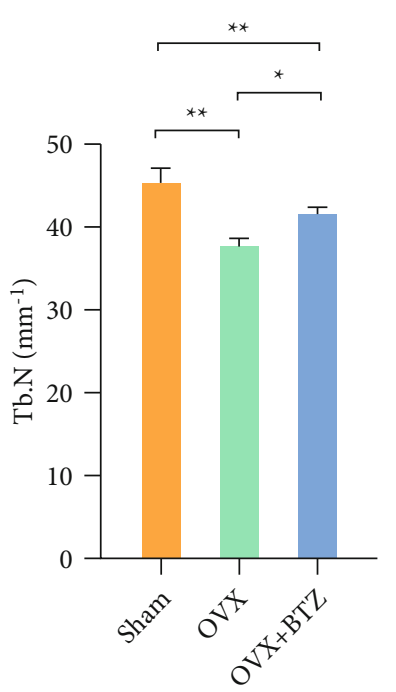

(d)

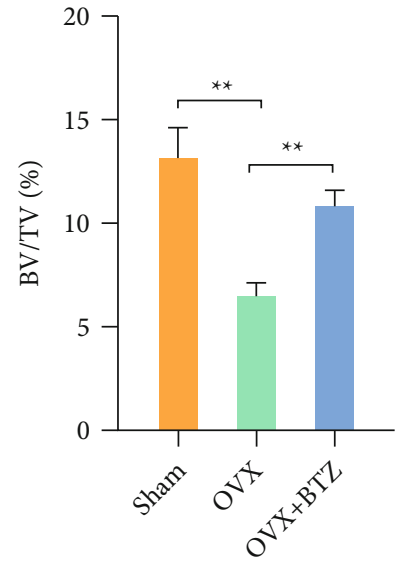

(e)

FIgURE 4: The effect of bortezomib on the radiographic bone loss in OVX mice. (a) Representative images of micro-CT reconstruction for coronal and cross-sectional plane of mice femurs. (b) Quantification of bone microstructure parameters including bone mineral density $(\mathrm{BMD})$, the bone volume ratio (BV/TV, \%), bone surface/volume ratio (BS/BV, $\mathrm{mm}^{-1}$ ), and trabecular number $\left(\mathrm{Tb} . \mathrm{N}, \mathrm{mm}{ }^{-1}\right)$. Values represent mean $\pm \mathrm{SD}$ of six samples in each group $(n=6)$ in micro-CT assays. Statistically significant differences are indicated by $*$ where $p<0.05$ or $* *$ where $p<0.01$ between the indicated groups.

that bortezomib promoted osteoblast differentiation in MM patients [27].

Since bortezomib exhibited greater potential for treating $\mathrm{OP}$, we analyzed its effects on osteogenesis and osteoblastogenesis (Figure 7). Accordingly, we found that $1 \mathrm{nM}$ of bortezomib enhanced the osteogenic potential of MC3T3-E1 cells during the early (7 days) and late (14 days) induction periods. A previous study has reported a stimulating effect of in vitro bortezomib on osteoblast differentiation [28]. In our study, bortezomib upregulated the levels of osteoprotegerin (OPG) and runt-related transcription factor 2 (RUNX2), two genes involved in osteoblast differentiation. OPG reportedly acts as an inhibitor of osteoclast differentiation by disturbing the RANK/RNAKL activation pathway [29]. In this regard, OPG-knockout (KO) mice exhibited severe osteoporosis with over-activated osteoclast formation [30]. Moreover, RUNX2 has been reported to be a master modulator of osteoclast differentiation. Expression of RUNX2 has been associated with cranial suture closure and membranous bone morphogenesis at the embryonic stage [31]. Furthermore, postnatal global deletion of RUNX2 can result in bone loss and excessive bone marrow adiposity in mice bone tissues [32].

In addition to promoting osteogenesis, we revealed that the same concentration of bortezomib inhibited osteoclast differentiation in BMM osteoclast precursors. Consistent with the reduced number of TRAP-positive multinucleated osteoclasts, differentially expressed proteins (e.g., TRAP, MMP9, CTSK, and NFATc1) involved in osteoclastogenesis were downregulated by bortezomib. Moreover, $2 \mathrm{nM}$ of bortezomib was reported to achieve similar findings in another study [13], corroborating that bortezomib can induce potent antiosteoclastogenesis effects. However, the mechanisms underlying bortezomib-mediated osteoclastogenesis inhibi- tion are unknown and warrant further studies. Much controversy surrounds several aspects (e.g., dosage and drugdelivery methods) essential for the in vivo application of bortezomib in animal models. Although bortezomib has exhibited good clinical outcomes for MM treatment, it has been associated with several adverse events, e.g., peripheral neuropathy, cardiovascular toxicity, and skeletal muscle weakness [33]. Intraperitoneal injection of bortezomib for 30 days suppressed the growth and invasion of chondrosarcoma in BALB/c athymic nude mouse osteosarcoma model in a study by Bao et al. [34]. Using a similar in vivo drug delivery method, we found bortezomib exerted a protective effect on bone loss in C57BL/6J mice. Considering the adverse effects associated with bortezomib, including the potential risk for other organ systems, and determining which method of drug administration (local or systemic) may be more beneficial, Wang et al. performed intraarticular injections of bortezomib $(0.5 \mathrm{mg} / \mathrm{mL})$ in posttraumatic OA mice, which showed a decreased joint tissue damage and reduced inflammatory levels [35]. Interestingly, a novel tumor microenvironment-targeted nanoparticle loaded with bortezomib was designed to target the MMassociated endothelium and synchronize drug delivery to enhance clinical outcomes in $\mathrm{MM}$ and reduce the side effects [36]. Moreover, in another study, bortezomibloaded nanomedicine assembled by $\mathrm{pH}$-responsive properties showed minimal cytotoxicity at near-neutral $\mathrm{pH}$ and exerted an enhanced anticancer activity for metastatic bone tumors [37].

Herein, we performed bioinformatic analyses and sought to unravel the mechanisms underlying bortezomib modulation of osteoblast and osteoclast differentiation. SMURFmediated ubiquitination pathway was found to be involved in the protective effect exerted by bortezomib against 

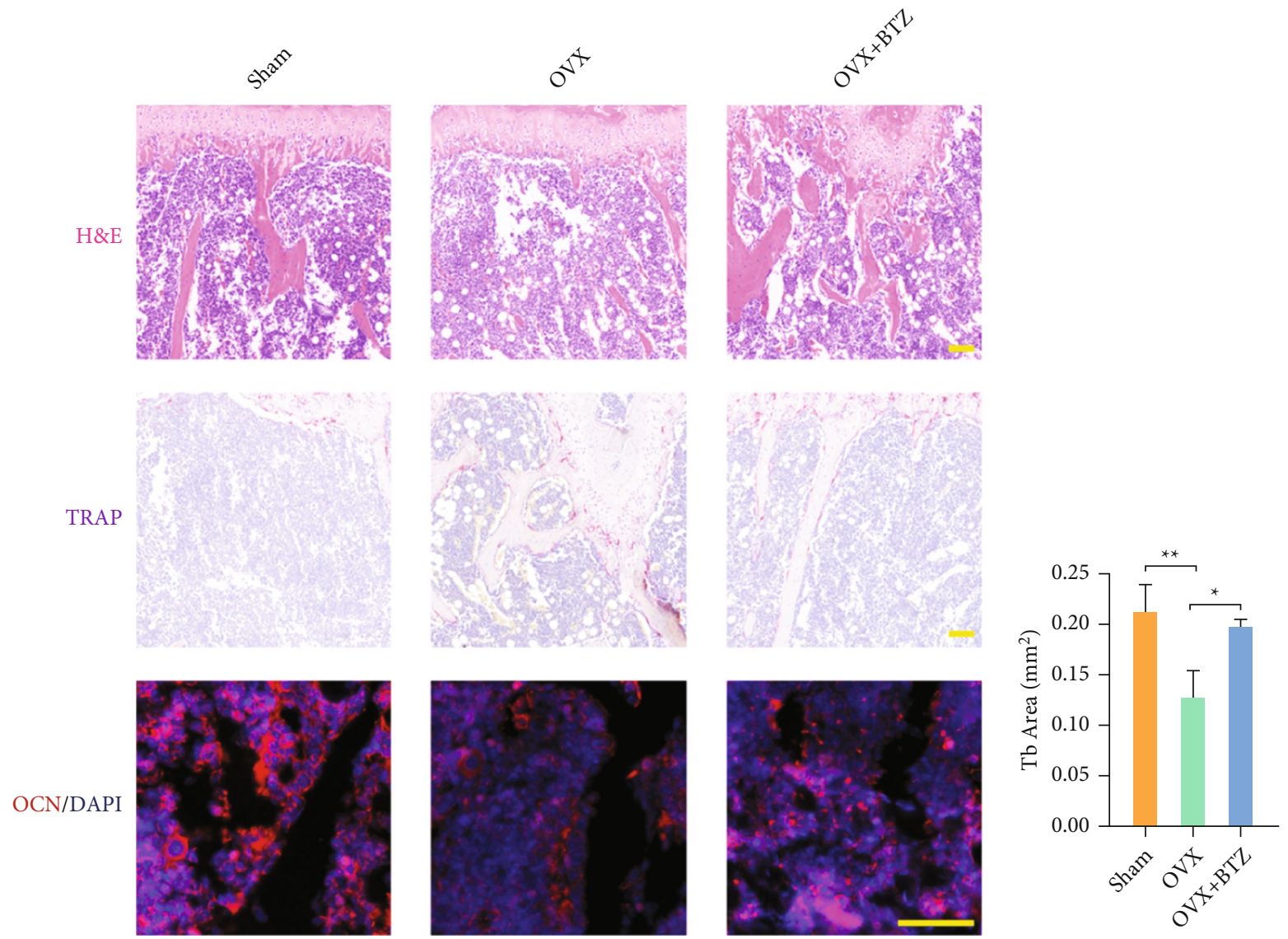

(a)

(b)

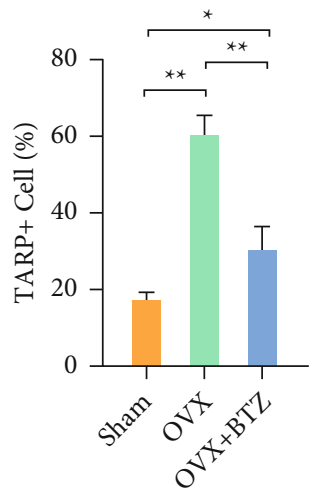

(c)

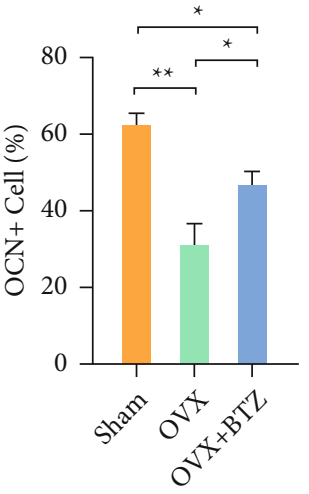

(d)

FIgURE 5: The effect of bortezomib on the histological bone loss in OVX mice. (a) Representative histological images of rat femurs stained by hematoxylin and eosin (H\&E), tartrate-resistant acid phosphatase (TRAP), and OCN immunofluorescence staining. Scale bar $=100 \mu \mathrm{m}$. (b) Quantification of bone microstructure parameters including Tb area. (c, d) The percentage of the number of TRAP- or OCN-positive cells. Values represent mean \pm SD of six samples in each group $(n=6)$ in histological staining assays. Statistically significant differences are indicated by $*$ where $p<0.05$ or $* *$ where $p<0.01$ between the indicated groups.

postmenopausal OP. Overexpression of SMURF2 was documented to delay fracture healing by modulating the ubiquitin level of the $\mathrm{Wnt} / \beta$-catenin signaling pathway of in vivo femur fracture models [38]. Additionally, in an aginginduced intervertebral disc degeneration (IVDD) mice model, ectopic expression of SMURF2 accelerated proteoglycan loss and collagen fibrosis of nucleus pulposus (NP) [39]. Intriguingly, osteoblasts derived from OP patients decreased by $81.2 \%$ compared with control patients, which seems contrary to the negative role of SMURF2 in OP. We hypothesized that such contradiction might be attributed to confounding factors such as different OP stages and types. Intriguingly, beyond SMURF2, RGS4 and HEY2 were identified as the potential hub-genes. A latest study indicated that the expression of RGS4 was up-regulated during the osteogenic induction of adipose-derived stem cell (ADSC). 


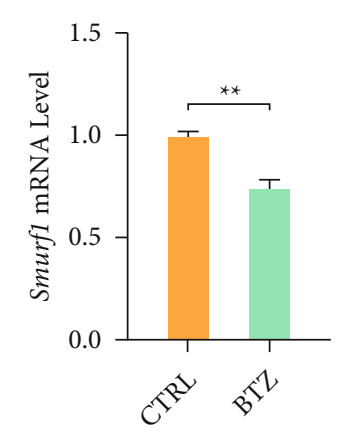

(a)

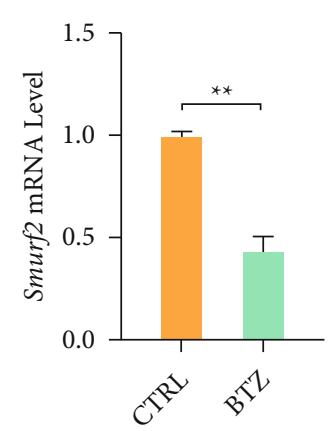

(b)
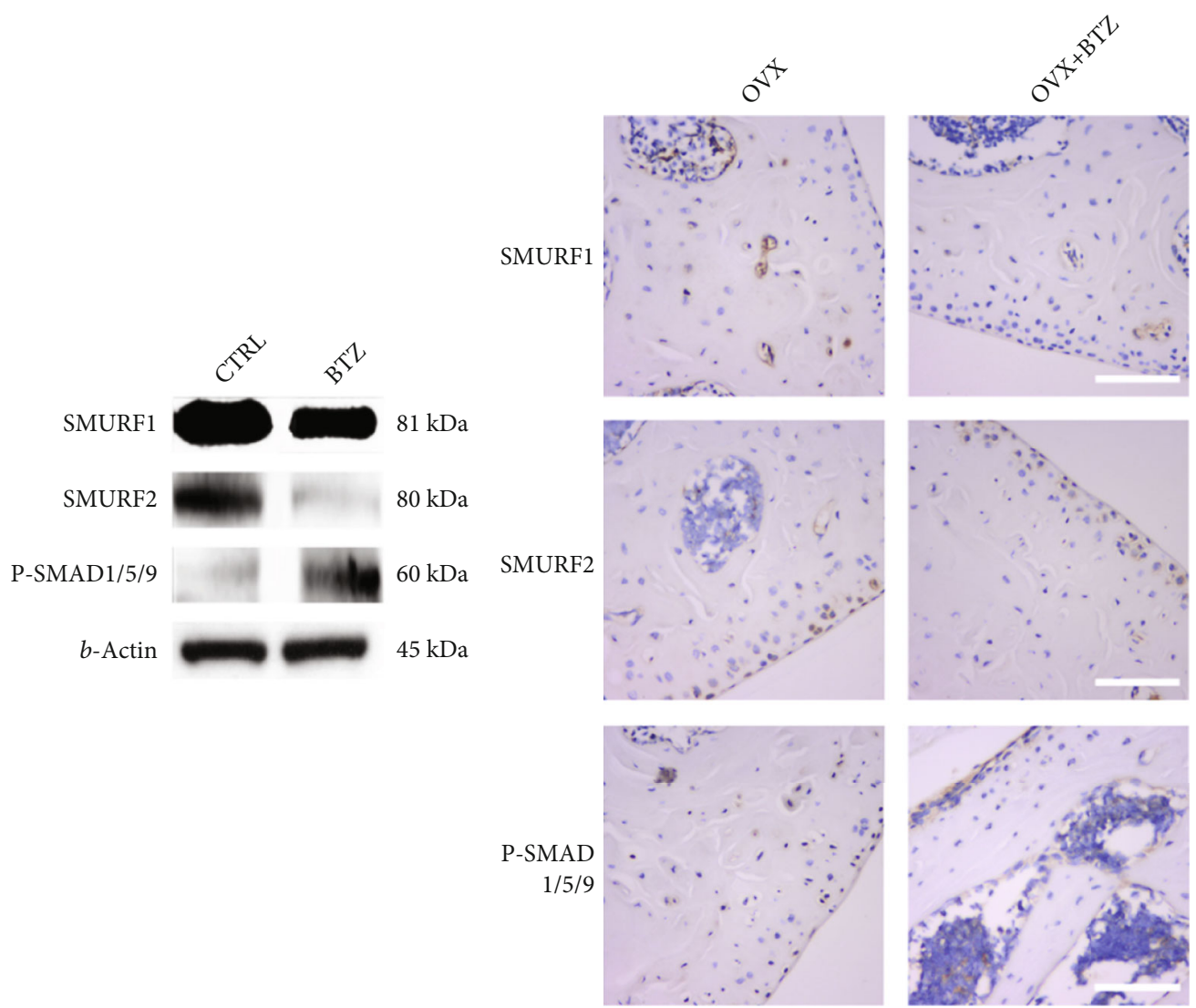

(d)

Figure 6: Continued. 


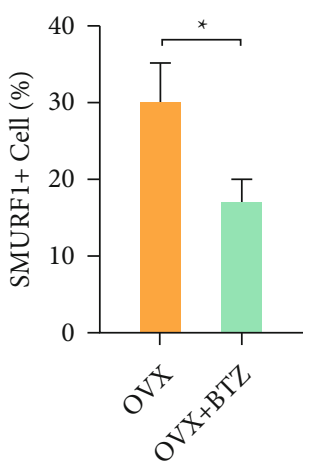

(e)

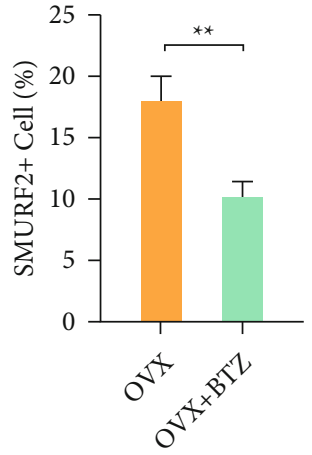

(f)

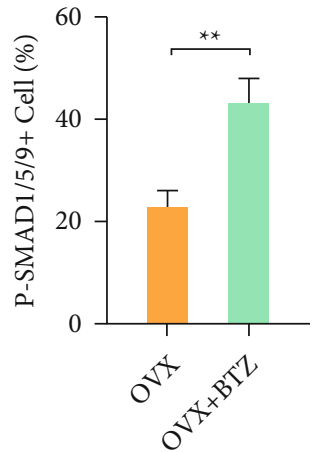

(g)

FIGURE 6: The involvement of SMURF1/2 in the bortezomib-mediated antiosteoporotic effect. (a, b) The mRNA levels of smurf1 and smurf2 genes were quantified with real-time RT-PCR using GAPDH as the normalization. (c) The protein levels of SMURF enzymes and SMAD pathway were determined using Western blot assays. (d) Representative immunohistology images stained by SMURF1, SMURF2, and pSMAD1/5/9. (e-g) Quantification of the cell number of SMURF1-, SMURF2-, or P-SMAD-1/5/9-positive cells. Values represent mean \pm SD of six samples in each group $(n=6)$ in histological staining assays. Statistically significant differences are indicated by $*$ where $p<$ 0.05 or $* *$ where $p<0.01$ between the indicated groups.

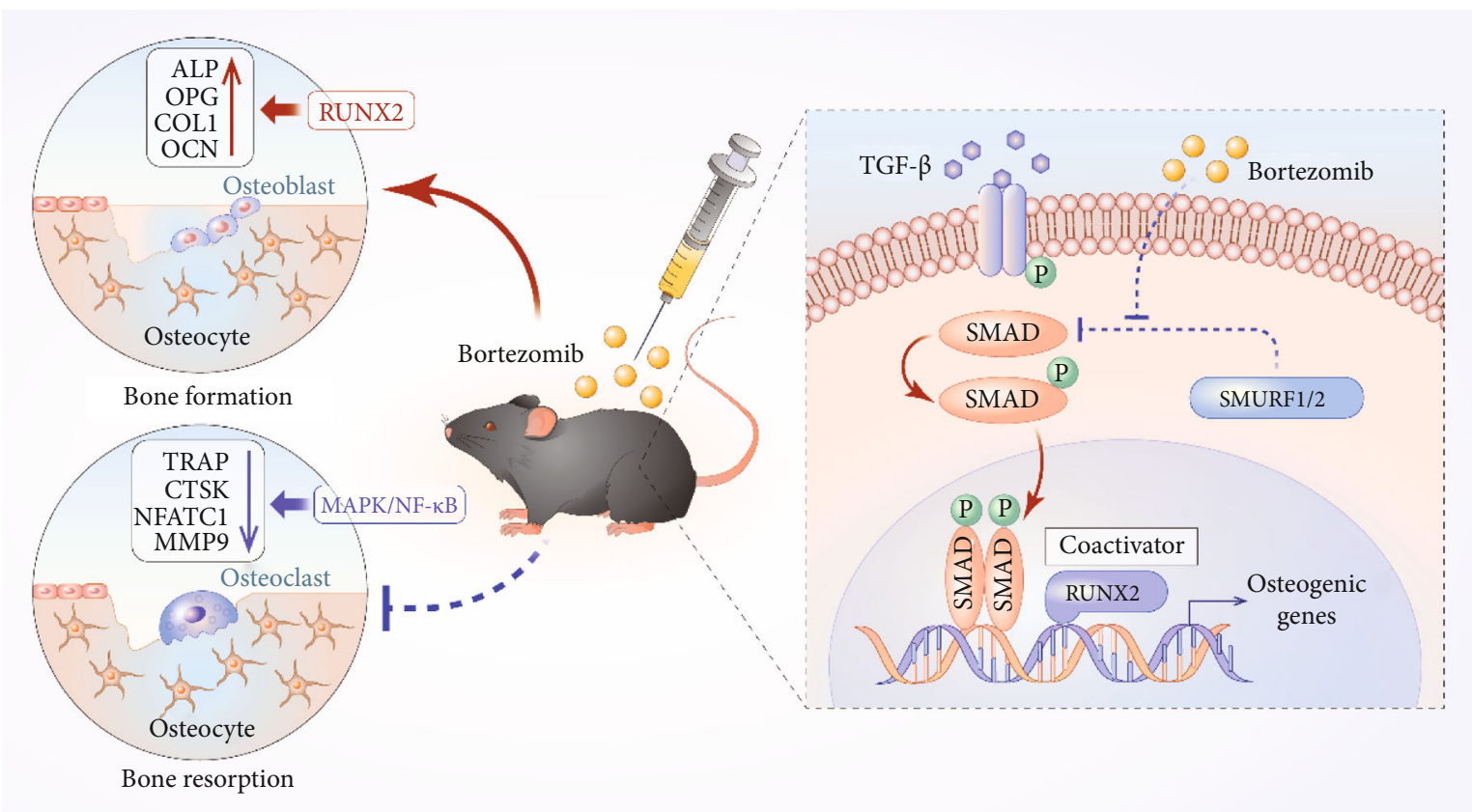

Figure 7: In vivo administration of bortezomib alleviates OVX-induced bone loss in mice. Bortezomib exerts a dual effect on promoting osteogenesis and suppressing osteoclastogenesis in vitro. Mechanically, bortezomib-mediated antiosteoporotic effect depends on the SMURF-related ubiquitination pathway.

Overexpression of LINC00370 sponge miR-222-3p to enhance RGS4-medicated bone protection [40]. Conversely, as a negative regulator, suppressing HEY2 with Notch2 antisense oligonucleotides (ASO) attenuated the cancellous osteopenia of Notch2 $2^{\text {tm1.1Ecan }}$ mice [41]. The potential amphoteric effects of bortezomib on RGS4 and HEY2 in OP will be explored in our further work.

Furthermore, according to our results, bortezomibinduced inhibition of SMURF was associated with increased expression of SMAD3. Xu et al. revealed that SMURF2 could modulate RANKL expression via blocking the interaction between SMAD3 and vitamin D receptor which was depen- dent on SMAD3 ubiquitination. Furthermore, SMURF2 can regulate osteoblast differentiation via ubiquitin/proteasomemediated degradation of RUNX2 [42]. Therefore, the detailed mechanisms of the bortezomib-induced OPantagonistic effect through SMURF warrants further exploration. Furthermore, multiple miRNAs, e.g., miR-615-3p [43], miR-30a-3p [44], miR-29b [45], and miR-324-5p [46], were found to be implicated in bortezomib-exerted anti-MM effect. The involvement of noncoding RNA will be further explored in our future work. Several limitations should be emphasized in this study. First, we only built an estrogen-deficient OP model; an aging-induced model was 
not studied due to time constraints. Second, further activation or knockdown experiments of SMURF are essential to improve the robustness of our findings and will be carried out in future studies. Meanwhile, the MC3T3-E1 cell line but not primary cells was studied in this experiment. In the subsequent experiment, we will investigate the salutary effects of bortezomib on rat-derived BMMSC and osteoporotic rat-derived BMMSC.

\section{Conclusions}

We demonstrated that bortezomib successfully enhanced the osteogenic potential and modulated osteoclastogenesis to regulate bone homeostasis in vitro. Furthermore, intraperitoneal administration of bortezomib improved the bone mineral density and microarchitecture in OVX-induced mice femurs in vivo. The bortezomib-mediated antiosteoporotic effect was achieved via the SMURF-mediated ubiquitination pathway. Accordingly, our findings substantiate that bortezomib may be a potential drug for treating osteoporosis.

$\begin{array}{ll}\text { Abbreviations } \\ \text { BTZ: } & \text { Bortezomib } \\ \text { MM: } & \text { Multiple myeloma } \\ \text { OP: } & \text { Osteoporosis } \\ \text { OVX: } & \text { Ovariectomized } \\ \text { BMMs: } & \text { Bone marrow monocytes } \\ \text { TRAP: } & \text { Tartrate-resistant acid phosphatase } \\ \text { SMURF: } & \text { Smad ubiquitination regulatory factor } \\ \text { OVCF: } & \text { Osteoporotic vertebral compression fracture } \\ \text { RA: } & \text { Rheumatoid arthritis } \\ \text { UPS: } & \text { Ubiquitin-proteasome system } \\ \text { TGF: } & \text { Transforming growth factor } \\ \text { BMP: } & \text { Bone morphogenetic protein } \\ \text { FBS: } & \text { Fetal bovine serum } \\ \text { RBC: } & \text { Red blood cells } \\ \text { M-CSF: } & \text { Macrophage-colony stimulating factor } \\ \text { RANKL: } & \text { Receptor activator of nuclear factor- } \kappa \text { B ligand } \\ \text { PBS: } & \text { Phosphate-buffered saline } \\ \text { BMMSCs: } & \text { Bone marrow stem cells } \\ \text { OPG: } & \text { Osteoprotegerin } \\ \text { IVDD: } & \text { Intervertebral disc degeneration } \\ \text { NP: } & \text { Nucleus pulposus. } \\ & \end{array}$

\section{Data Availability}

The data used to support the findings of this study are available from the corresponding authors upon request.

\section{Conflicts of Interest}

The authors declare that they have no conflict of interest.

\section{Authors' Contributions}

Yuepeng Fang, Yijian Zhang, and Xuesong Zhu designed the research study; Yuepeng Fang, Yijian Zhang, Yang Liu, Zhijian Zhao, Yingjie Lu, Xu Shen, Tianfeng Zhu, and Min- gzhuang Hou performed the experiments; Yuepeng Fang, Yijian Zhang, Qin Shi, and Xuesong Zhu analyzed the data; Yijian Zhang and Xuesong Zhu wrote the paper; and Fan He, Huilin Yang, and Fan He participated in the study's conception and revision of the manuscript. All authors approve of the final version to be published. Yuepeng Fang, Yang Liu, Zhijian Zhao, and Yingiie Lu contributed equally to this work.

\section{Acknowledgments}

This work was supported by the National Natural Science Foundation of China (31771063, 81702146, 81772358, 82072442), the Priority Academic Program Development of Jiangsu Higher Education Institutions (PAPD), and the Postgraduate Research \& Practice Innovation Program of Jiangsu Province (KYCX21_2971).

\section{Supplementary Materials}

Supplementary Figure 1: quantification of the protein levels of osteogenic markers in bortezomib-treated MC3T3-E1 cells. Supplementary Figure 2: quantification of the bone resorption indicators and protein levels of osteoclastogenic markers in bortezomib-treated BMM cells. Supplementary Figure 3: quantification of the protein levels of ubiquitination markers in bortezomib-treated MC3T3-E1 cells. (Supplementary Materials)

\section{References}

[1] N. Sarafrazi, E. A. Wambogo, and J. A. Shepherd, "Osteoporosis or low bone mass in older adults: United States, 20172018," NCHS Data Brief, vol. 405, pp. 1-8, 2021.

[2] X. Cheng, K. Zhao, X. Zha et al., "Opportunistic screening using low-dose CT and the prevalence of osteoporosis in China: a nationwide multicenter study," Journal of Bone and Mineral Research, vol. 36, no. 3, pp. 427-435, 2021.

[3] H. Yang, L. Chen, Z. Zheng et al., "Therapeutic effects analysis of percutaneous kyphoplasty for osteoporotic vertebral compression fractures: a multicentre study," Journal of Orthopaedic Translation, vol. 11, pp. 73-77, 2017.

[4] Y. Zhang, F. He, H. Yang, and X. Zhu, "Deletion of SIRT3 inhibits osteoclastogenesis and alleviates aging or estrogen deficiency-induced bone loss in female mice," Bone, vol. 151, article 116061, 2021.

[5] W. Yu, L. Zhong, L. Yao et al., "Bone marrow adipogenic lineage precursors promote osteoclastogenesis in bone remodeling and pathologic bone loss," The Journal of Clinical Investigation, vol. 131, no. 2, 2021.

[6] K. Scott, P. J. Hayden, A. Will, K. Wheatley, and I. Coyne, "Bortezomib for the treatment of multiple myeloma," Cochrane Database of Systematic Reviews, vol. 4, article Cd010816, 2016.

[7] I. T. Huang, B. Dhungel, R. Shrestha et al., "Spotlight on bortezomib: potential in the treatment of hepatocellular carcinoma," Expert Opinion on Investigational Drugs, vol. 28, no. 1, pp. 7-18, 2019.

[8] W. Hu, W. Zhang, F. Li, F. Guo, and A. Chen, "Bortezomib prevents the expression of MMP-13 and the degradation of collagen type 2 in human chondrocytes," Biochemical and 
Biophysical Research Communications, vol. 452, no. 3, pp. 526-530, 2014.

[9] S. Lassoued, C. Moyano, M. Beldjerd, P. Pauly, D. Lassoued, and T. Billey, "Bortezomib improved the joint manifestations of rheumatoid arthritis in three patients," Joint, Bone, Spine, vol. 86, no. 3, pp. 381-382, 2019.

[10] Z. Zhang, X. Fu, L. Xu et al., "Nanosized Alumina Particle and Proteasome Inhibitor Bortezomib Prevented inflammation and Osteolysis Induced by Titanium Particle via Autophagy and NF- $\kappa$ B Signaling," Scientific Reports, vol. 10, no. 1, p. 5562, 2020.

[11] M. Mohty, F. Malard, B. Mohty, B. Savani, P. Moreau, and E. Terpos, "The effects of bortezomib on bone disease in patients with multiple myeloma," Cancer, vol. 120, no. 5, pp. 618-623, 2014.

[12] M. I. Suominen, J. Mäki-Jouppila, A. Huhtinen et al., “Additive benefits of radium-223 dichloride and bortezomib combination in a systemic multiple myeloma mouse model," International Journal of Molecular Sciences, vol. 22, no. 11, p. 5570, 2021.

[13] S. H. Kim, M. O. Kim, H. J. Kim et al., "Bortezomib prevents ovariectomy-induced osteoporosis in mice by inhibiting osteoclast differentiation," Journal of Bone and Mineral Metabolism, vol. 36, no. 5, pp. 537-546, 2018.

[14] D. Popovic, D. Vucic, and I. Dikic, "Ubiquitination in disease pathogenesis and treatment," Nature Medicine, vol. 20, no. 11, pp. 1242-1253, 2014.

[15] S. Tsuchida, M. Satoh, M. Takiwaki, and F. Nomura, "Ubiquitination in periodontal disease: a review," International Journal of Molecular Sciences, vol. 18, no. 7, p. 1476, 2017.

[16] M. Datto and X. F. Wang, "Ubiquitin-Mediated Degradation: A Mechanism for Fine-TuningTGF- $\beta$ Signaling," Cell, vol. 121, no. 1, pp. 2-4, 2005.

[17] S. X. Ying, Z. J. Hussain, and Y. E. Zhang, "Smurf1 Facilitates Myogenic Differentiation and Antagonizes the Bone Morphogenetic Protein-2-induced Osteoblast Conversion by Targeting Smad5 for Degradation*," The Journal of Biological Chemistry, vol. 278, no. 40, pp. 39029-39036, 2003.

[18] J. Kushioka, T. Kaito, R. Okada et al., "A novel negative regulatory mechanism of Smurf2 in BMP/Smad signaling in bone," Bone Research, vol. 8, no. 1, p. 41, 2020.

[19] Z. Wang, J. Wang, X. Li et al., "Bortezomib prevents oncogenesis and bone metastasis of prostate cancer by inhibiting WWP1, Smurf1 and Smurf2," International Journal of Oncology, vol. 45, no. 4, pp. 1469-1478, 2014.

[20] M. Li, X. Chen, J. Yan et al., "Inhibition of osteoclastogenesis by stem cell-derived extracellular matrix through modulation of intracellular reactive oxygen species," Acta Biomaterialia, vol. 71, pp. 118-131, 2018.

[21] Y. Zhang, J. Lin, X. Zhou et al., "Melatonin prevents osteoarthritis-induced cartilage degradation via targeting microRNA-140," Oxidative Medicine and Cellular Longevity, vol. 2019, Article ID 9705929, 16 pages, 2019.

[22] W. Yang, X. Lu, T. Zhang et al., "TAZ inhibits osteoclastogenesis by attenuating TAK1/NF- $\kappa \mathrm{B}$ signaling," Bone Research, vol. 9, no. 1, p. 33, 2021.

[23] X. Chen, M. Li, J. Yan et al., "Alcohol induces cellular senescence and impairs osteogenic potential in bone marrowderived mesenchymal stem cells," Alcohol and Alcoholism, vol. 52, no. 3, pp. 289-297, 2017.
[24] E. G. Estell and C. J. Rosen, "Emerging insights into the comparative effectiveness of anabolic therapies for osteoporosis," Nature Reviews. Endocrinology, vol. 17, no. 1, pp. 31-46, 2021.

[25] P. G. Richardson, A. Oriol, M. Beksac et al., "Pomalidomide, bortezomib, and dexamethasone for patients with relapsed or refractory multiple myeloma previously treated with lenalidomide (OPTIMISMM): a randomised, open-label, phase 3 trial," The Lancet Oncology, vol. 20, no. 6, pp. 781-794, 2019.

[26] C. Shimazaki, R. Uchida, S. Nakano et al., "High serum bonespecific alkaline phosphatase level after bortezomib- combined therapy in refractory multiple myeloma: possible role of bortezomib on osteoblast differentiation," Leukemia, vol. 19, no. 6, pp. 1102-1103, 2005.

[27] N. Giuliani, F. Morandi, S. Tagliaferri et al., "The proteasome inhibitor bortezomib affects osteoblast differentiation in vitro and in vivo in multiple myeloma patients," Blood, vol. 110, no. 1, pp. 334-338, 2007.

[28] H. Wang, H. Zhang, V. Srinivasan et al., "Targeting bortezomib to bone increases its bone anabolic activity and reduces systemic adverse effects in mice," Journal of Bone and Mineral Research, vol. 35, no. 2, pp. 343-356, 2020.

[29] D. L. Lacey, W. J. Boyle, W. S. Simonet et al., "Bench to bedside: elucidation of the OPG-RANK-RANKL pathway and the development of denosumab," Nature Reviews. Drug Discovery, vol. 11, no. 5, pp. 401-419, 2012.

[30] N. Bucay, I. Sarosi, C. R. Dunstan et al., "Osteoprotegerin-deficient mice develop early onset osteoporosis and arterial calcification," Genes \& Development, vol. 12, no. 9, pp. 1260-1268, 1998.

[31] K. Y. Choi, S. W. Lee, M. H. Park et al., "Spatio-temporal expression patterns of Runx 2 isoforms in early skeletogenesis," Experimental \& Molecular Medicine, vol. 34, no. 6, pp. 426433, 2002.

[32] I. Tosa, D. Yamada, M. Yasumatsu et al., "Postnatal Runx2 deletion leads to low bone mass and adipocyte accumulation in mice bone tissues," Biochemical and Biophysical Research Communications, vol. 516, no. 4, pp. 1229-1233, 2019.

[33] E. Pancheri, V. Guglielmi, G. M. Wilczynski et al., "Nonhematologic toxicity of bortezomib in multiple myeloma: the neuromuscular and cardiovascular adverse effects," Cancers, vol. 12, no. 9, p. 2540, 2020.

[34] X. Bao, T. Ren, Y. Huang et al., "Bortezomib induces apoptosis and suppresses cell growth and metastasis by inactivation of Stat3 signaling in chondrosarcoma," International Journal of Oncology, vol. 50, no. 2, pp. 477-486, 2017.

[35] W. Wang, X. Lin, H. Xu et al., “Attenuated joint tissue damage associated with improved synovial lymphatic function following treatment with bortezomib in a mouse model of experimental posttraumatic osteoarthritis," Arthritis \& Rhematology, vol. 71, no. 2, pp. 244-257, 2019.

[36] C. Federico, K. Alhallak, J. Sun et al., "Tumor microenvironment-targeted nanoparticles loaded with bortezomib and ROCK inhibitor improve efficacy in multiple myeloma," Nature Communications, vol. 11, no. 1, p. 6037, 2020.

[37] M. Wang, X. Cai, J. Yang et al., "A targeted and $\mathrm{pH}$-responsive bortezomib nanomedicine in the treatment of metastatic bone tumors," ACS Applied Materials \& Interfaces, vol. 10, no. 48, pp. 41003-41011, 2018.

[38] Y. Huang, Y. Xu, S. Feng, P. He, B. Sheng, and J. Ni, “miR-19b enhances osteogenic differentiation of mesenchymal stem cells and promotes fracture healing through the WWP1/Smurf2- 
mediated KLF5/ $\beta$-catenin signaling pathway," Experimental \& Molecular Medicine, vol. 53, no. 5, pp. 973-985, 2021.

[39] Q. Wu and J. H. Huang, "Ectopic expression of Smurf2 and acceleration of age-related intervertebral disc degeneration in a mouse model," Journal of Neurosurgery. Spine, vol. 27, no. 1, pp. 116-126, 2017.

[40] L. Li, B. Zheng, F. Zhang et al., "LINC00370 modulates miR222-3p-RGS4 axis to protect against osteoporosis progression," Archives of Gerontology and Geriatrics, vol. 97, article 104505, 2021.

[41] E. Canalis, T. R. Grossman, M. Carrer, L. Schilling, and J. Yu, "Antisense oligonucleotides targeting _Notch2_ ameliorate the osteopenic phenotype in a mouse model of HajduCheney syndrome," The Journal of Biological Chemistry, vol. 295, no. 12, pp. 3952-3964, 2020.

[42] Z. Xu, M. B. Greenblatt, G. Yan et al., "SMURF2 regulates bone homeostasis by disrupting SMAD3 interaction with vitamin D receptor in osteoblasts," Nature Communications, vol. 8, no. 1, p. 14570, 2017.

[43] J. Liu, F. Du, C. Chen et al., "CircRNA ITCH increases bortezomib sensitivity through regulating the miR-615-3p/PRKCD axis in multiple myeloma," Life Sciences, vol. 262, article 118506, 2020.

[44] F. Nian, J. Zhu, and H. Chang, "Long non-coding RNA ANGPTL1-3 promotes multiple myeloma bortezomib resistance by sponging miR-30a-3p to activate c-Maf expression," Biochemical and Biophysical Research Communications, vol. 514, no. 4, pp. 1140-1146, 2019.

[45] Y. Fu, X. Liu, F. Zhang, S. Jiang, J. Liu, and Y. Luo, “Bortezomib-inducible long non-coding RNA myocardial infarction associated transcript is an oncogene in multiple myeloma that suppresses miR-29b," Cell Death \& Disease, vol. 10, no. 4, p. 319, 2019.

[46] B. Tang, A. Xu, J. Xu et al., "MicroRNA-324-5p regulates stemness, pathogenesis and sensitivity to bortezomib in multiple myeloma cells by targeting hedgehog signaling," International Journal of Cancer, vol. 142, no. 1, pp. 109-120, 2018. 